\title{
Best Poster Award
}

Topic: 5. Secondary Prevention of Cardiovascular Disease.

\section{Oral Anticoagulation and Prognosis of Patients with Atrial Fibrillation After Ischemic Stroke: A Real-world Data Analysis}

\author{
Mario Diaznuila-Alcázar, Daniel Valcárcel-Paz, Pablo Del Castillo-Vásquez, Marcel Santaló-Corcoy,
} Walter Bragagnini, Joan Carles Oliva-Morera, Eduard Bosch-Peligero, Antoni Martínez-Rubio

Keywords: Atrial fibrillation, secondary prevention

\section{Introduction}

Atrial fibrillation (AF) is one of the most common arrhythmias and a recognized source of embolic ischemic stroke which has critical prognostic implications.

\section{Objetives}

To prospectively analyze the clinical characteristics of hospitalized patients with atrial fibrillation presenting with an ischemic stroke, the subsequent preventive strategies, the quality of dicumarinic therapy (if used) and the posterior outcomes (cardiovascular events and mortality).

\section{Materials and Methods}

Between January of 2006 and June 2013, 370 patients (pts) (Age: $81+$ 7 years, $66 \%$ female, $\mathrm{CHA}_{2} \mathrm{DS}_{2}$-VASC: $5,1+1,7$, arterial hypertension: $80 \%$, chronic kidney disease (CKD): $40 \%$, diabetics: $28 \%$, dyslipemics: $34 \%$, any prior bleeding: $19 \%$, previous ischemic stroke: $9 \%$, chronic obstructive pulmonary disease: $7 \%$ ) were admitted to the hospital because of stroke and $\mathrm{AF}$ and form the basis of analysis.

\section{Results}

From those 370 pts, 72 pts (19 \%) already died in- hospital during the index event. Based on the decision of the attending physicians (who considered the clinical status and characteristics of those individuals who survived the index event $(n=298)$ ) and the chosen treatment, four subgroups of strategies were identified: Dicoumarin therapy (DIC), direct oral anticoagulation (DOAC), antiplatelet therapy (APT), finally without APT and without anticoagulant therapy (NO-T).

The DIC subgroup was composed of 129/298 pts (43 \%). Furthermore, during a follow-up of 22+18 months, 4270 INR level determinations were performed (mean number of INR determinations per patient was $30 \pm 18)$. A total of 1879 INR determinations (44 \%) showed an inappropriate level $(<2$ or $>3$ ). Thus, overall patients were during $7.9 \pm 6$ months at risk of thromboembolic or bleeding events in relationship to INR out of therapeutic range. The DOAC, APT and NO-T groups included 25 (8\%), 112 (37\%) and 32 (11\%) pts, respectively.

The follow-up of survivors lasted until June 2016. At this date, only 90 (24\%) pts were alive (total death rate of $76 \%$ ). Cardiovascular mortality occurred in 162/280 (58 \%) of deceased patients.

\section{Conclusions}

A population with AF suffering ischemic stroke, advanced age and a high-risk profile showed a very poor outcome (reflected by a very high mortality). Even in an identified very high-risk population, the DIC therapy shows an inappropriate INR in $44 \%$ of the determinations. Therefore, modern therapies (e.g. DOAC) should be more often used. 